\title{
Disentangling Cooper-pair formation above the transition temperature from the pseudogap state in the cuprates
}

\author{
Takeshi Kondo ${ }^{1 \star}$, Yoichiro Hamaya ${ }^{2}$, Ari D. Palczewski ${ }^{1}$, Tsunehiro Takeuchi ${ }^{2,3}$, J. S. Wen ${ }^{4}$, Z. J. Xu ${ }^{4}$, \\ Genda Gu${ }^{4}$, Jörg Schmalian ${ }^{1}$ and Adam Kaminski ${ }^{1 \star}$
}

The discovery of the pseudogap in the cuprates ${ }^{1-3}$ created significant excitement as it was believed to be a signature of pairing ${ }^{4}$, in some cases above room temperature. Indeed, a number of experiments detected phase-fluctuating superconductivity above the transition temperature $T_{c}$ (refs 5-9). However, several recent experiments reported that the pseudogap and superconducting state are characterized by different energy scales ${ }^{10-14}$, and probably compete with each other ${ }^{15,16}$, leaving open the question of whether the pseudogap is caused by pair formation. Here we report the discovery of a spectroscopic signature of pair formation and demonstrate that in a region commonly referred to as the pseudogap, two distinct states coexist: one that is due to pair formation and persists to an intermediate temperature $T_{\text {pair }}<T^{*}$ and a second-the 'proper' pseudogap-characterized by the loss of spectral weight and anomalies in transport properties that extends up to $T^{*}$. $T_{\text {pair }}$ has a value around $120-150 \mathrm{~K}$ even for materials with very different $T_{c}$ values and it probably sets a limit on the highest attainable $T_{c}$ in the cuprates.

The traditional approach of exploring the pairing above $T_{c}$ by tracking the energy scale of spectral features has so far not yielded convincing results, as these features are poorly defined above $T_{c}$. The apparent smooth evolution of the spectral gap from the lowest temperatures up to $T^{*}$ has previously been interpreted as key evidence for a common origin of the pseudogap and pairing gap ${ }^{17-20}$. However, very detailed, high-precision data demonstrate that the gap size actually does change across $T_{c}$ (as seen in Supplementary Fig. S3). Similar behaviour is indeed expected for a system with multiple gaps (in this case a pairing gap and pseudogap), when weights of the features associated with the respective gaps change with temperature. These data provide perhaps the strongest evidence supporting a 'two gaps' scenario in the cuprates. A better approach is to investigate the spectral weights, which are easier to quantify and interpret. A key measure of pair formation is the density of states at the Fermi energy $D\left(E_{\mathrm{F}}\right)$. In conventional, clean superconductors this weight is zero below $T_{c}$, but can be finite if there are strong impurity scattering effects. In such cases $D\left(E_{\mathrm{F}}\right)$ reflects the pair-breaking states. A generic 'density wave state' in the absence of pairing would also lead to a decrease of the $D\left(E_{\mathrm{F}}\right)$ due to the opening of the density wave gap. In addition there is also the possibility of the coexistence of superconductivity and the density wave state-inhomogeneous superconductors such as the cuprates $^{14,21-23}$, where superconducting and non-superconducting patches coexist in the sample. One can then expect that the temperature dependence of $D\left(E_{\mathrm{F}}\right)$ can be used to distinguish between these scenarios and disentangle the electronic ground states of the cuprates. In our study we use the intensity of the spectral function at $E_{\mathrm{F}}, I\left(E_{\mathrm{F}}, k\right)$, which when integrated over all momenta equals $D\left(E_{\mathrm{F}}\right)$, so the changes of one quantity with temperature are reflected in the other. This allows us to isolate the behaviour at a specific $k$-point and avoid smearing due to averaging. A discussion of the relation between $D\left(E_{\mathrm{F}}\right)$ and $I\left(E_{\mathrm{F}}, k\right)$ is provided in Supplementary Section S3.

In Fig. 1a-c we examine the temperature evolution of the spectral line shape, measured at the antinodal Fermi momentum in optimally doped $\operatorname{Bi2} 212\left(T_{\mathrm{c}}=90 \mathrm{~K}\right)$. Symmetrized energy distribution curves ${ }^{24}$ (EDCs) show the opening of the pseudogap on cooling below $T^{*}(\sim 210 \mathrm{~K})$. As the temperature is decreased below $T_{c}$, a sharp quasiparticle peak associated with superconductivity appears $^{25}$. We obtain the spectral changes with temperature by subtracting the spectrum at the highest temperature from all the spectra measured, as shown in Fig. 1b. Now we focus on the loss of spectral weight close to the Fermi level, $W\left(E_{\mathrm{F}}\right)$ (hatched area in Fig. 1b). The temperature dependence of $W\left(E_{\mathrm{F}}\right)$ is plotted in Fig. 1d. On cooling through $T^{*}$, the spectral weight decreases linearly, which is a characteristic behaviour of the pseudogap state. An astonishing feature seen in this plot is the clear deviation from linear behaviour (indicated by the arrow). As the temperature dependence below and above this point is very different, the arrow marks the onset of another distinct state. The onset temperature $T_{\text {pair }}(\sim 150 \mathrm{~K})$ of this transition is considerably higher than $T_{\mathrm{c}}(=90 \mathrm{~K})$, but is also significantly lower than the pseudogap temperature $T^{*}(\sim 210 \mathrm{~K})$. This state probably arises from pairing of the electrons because the weight loss associated with this state smoothly evolves through $T_{\mathrm{c}}$. If we extrapolate the linear variation of $W\left(E_{\mathrm{F}}\right)$ down to $T=0 \mathrm{~K}$, we obtain approximate values of the spectral weight lost as a result of the pseudogap, $W_{\mathrm{PG}}$ (blue area), and pairing, $W_{\text {pair }}$ (red area), as marked in Fig. $1 d$.

We now verify this hypothesis by studying how these quantities vary with doping in related samples of Bi2201, where $T_{\mathrm{c}}$ and $T^{*}$ are more separated over a wide range of carrier concentrations ${ }^{26}$. Figure $2 \mathrm{a}-\mathrm{g}$ shows symmetrized EDCs measured at the antinode for various temperatures and dopings from underdoped (left side)

\footnotetext{
${ }^{1}$ Ames Laboratory and Department of Physics and Astronomy, lowa State University, Ames, lowa 50011, USA, ${ }^{2}$ Department of Crystalline Materials Science, Nagoya University, Nagoya 464-8603, Japan, ${ }^{3}$ EcoTopia Science Institute, Nagoya University, Nagoya 464-8603, Japan, ${ }^{4}$ Condensed Matter Physics and Materials Science Department, Brookhaven National Laboratory, Upton, New York 11973, USA. *e-mail: kondo@ameslab.gov; kaminski@ameslab.gov.
} 


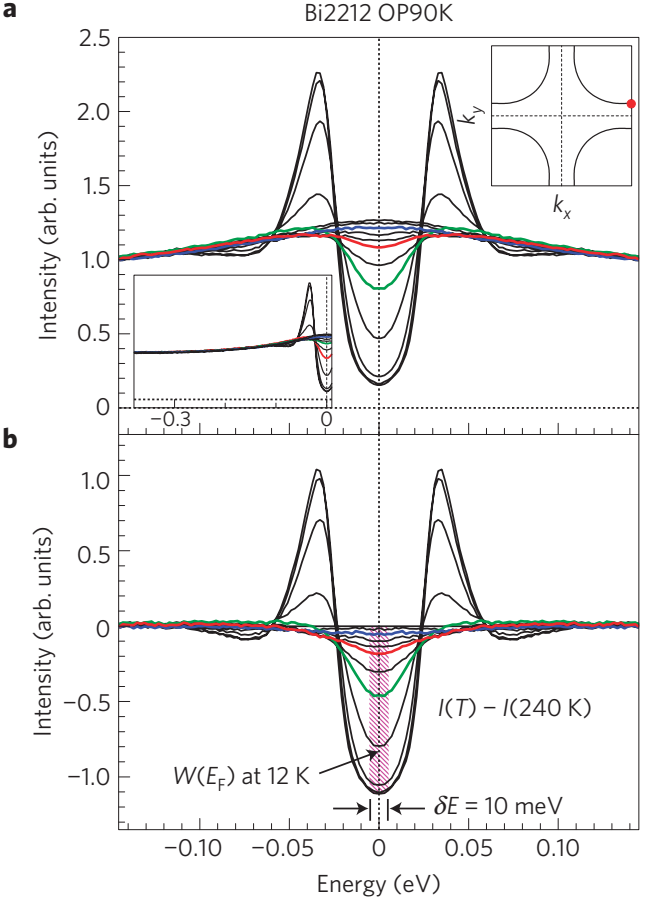

c

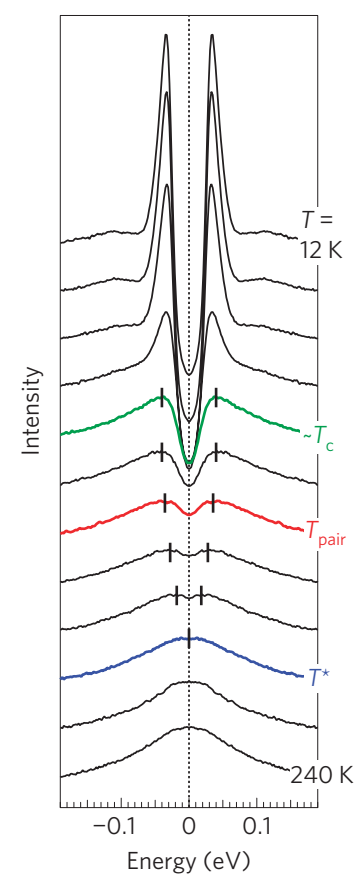

d

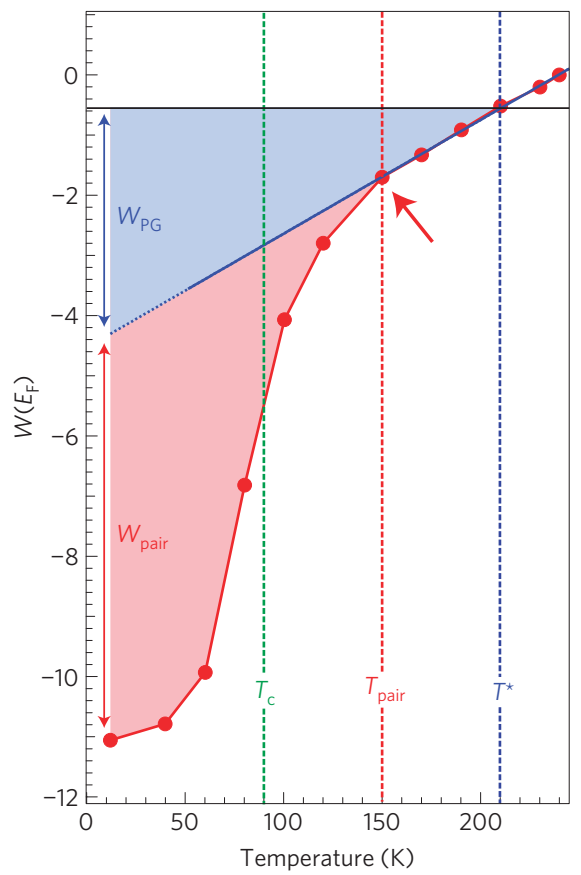

Figure 1 | Temperature dependence of the spectral weight at the Fermi energy. a, Symmetrized EDCs (ref. 24) for various temperatures from deep below $T_{\mathrm{c}}$ to above the pseudogap temperature $\left(T^{*}\right.$ ). The spectra were measured at the antinode (marked by the red point on the Fermi surface shown in the inset) in optimally doped $\mathrm{Bi} 2212\left(T_{\mathrm{C}}=90 \mathrm{~K}\right)$. b. Difference spectra. The spectrum measured at the highest temperature is subtracted from each of spectra in a. Spectral weight close to the Fermi level $\left(W\left(E_{\mathrm{F}}\right)\right.$, hatched area) is estimated by integrating the spectral intensity in $\mathbf{b}$ within an energy window of the

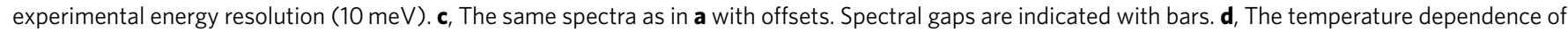
$W\left(E_{F}\right)$. The pairing temperature, $T_{\text {pair, }}$ is defined as the onset temperature of deviation (marked by arrow) from a linear behaviour seen at higher

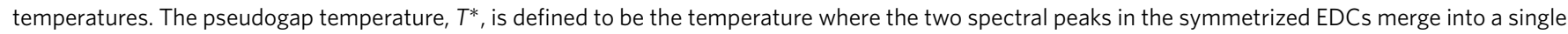
peak as seen in c. The three temperatures, $T_{c}$ (green), $T_{\text {pair }}$ (red) and $T^{*}$ (blue), are indicated with dashed lines. The pairing weight ( $W_{\text {pair, }}$ red area) and the pseudogap weight ( $W_{P G}$, blue area) are separated by a line extrapolated from the linear behaviour of $W\left(E_{F}\right)$ at high temperatures. Errors in EDC data

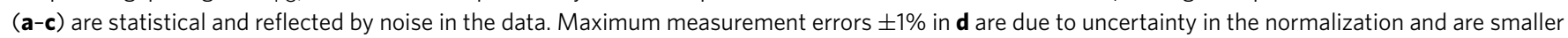
than data markers.

to overdoped (right side) samples. In Fig. 1c the peak positions are marked with short black lines to demonstrate that the spectral gap changes across $T_{c}$, as discussed in the second paragraph. The spectral changes of the EDCs with temperature are plotted in Fig. $2 \mathrm{~h}-\mathrm{n}$ and $W\left(E_{\mathrm{F}}\right)$ is plotted in Fig. 2o-u for all samples. As in the case of Bi2212 (Fig. 1), W(E $\left.E_{\mathrm{F}}\right)$ is linear below $T^{*}$ at high temperatures, then suddenly deviates from a straight line-defining a new temperature scale $T_{\text {pair }}$. The temperature dependence of $W\left(E_{\mathrm{F}}\right)$ evolves in a surprisingly systematic manner with doping. The linear part becomes longer with underdoping, as both $T^{*}$ and $T_{\text {pair }}$ increase. Eventually, at the lowest dopings, $W\left(E_{\mathrm{F}}\right)$ is linear down to the lowest temperature. This is because the quasiparticle residue vanishes at the antinode for heavily underdoped samples ${ }^{16}$ causing $W_{\text {pair }}$ to become very small. The $T_{\text {pair }}$ can be still extracted for such samples by examining $W\left(E_{\mathrm{F}}, T\right)$ slightly off the antinode. In case of UD23K $T_{\text {pair }}$ is $\sim 125 \mathrm{~K}$.

To validate our assertion about the pairing origin of $W_{\text {pair }}$, we extract this quantity for each doping by subtracting the interpolated $W_{\mathrm{PG}}$ line from each of the $W\left(E_{\mathrm{F}}, T\right)$ curves and compare them in Fig. 3a. Obviously the magnitude and onset temperature of $W_{\text {pair }}$ is very different for each doping. To make a fair comparison, we rescale the vertical axis for each curve by its maximum value at the lowest temperature and the horizontal axis by $T_{\text {pair }}$. Surprisingly the curves for all dopings fall on top of each other, demonstrating a universal scaling of $W_{\text {pair, }}$, which smoothly evolves through $T_{\mathrm{c}}$. Clearly, $W\left(E_{\mathrm{F}}, T\right)$ in the superconducting state reflects pair formation, which causes it to decrease on cooling. The temperature dependence of $W_{\text {pair }}$ does not exhibit significant features at $T_{\mathrm{c}}$ and its scaling remains unchanged across $T_{\mathrm{c}}$, strongly suggesting that pair formation occurs well above $T_{\mathrm{c}}$. As $W_{\text {pair }}$ scales with $T_{\text {pair }}$, this must be the onset temperature of pairing. At the lowest dopings (Fig. 2o), $W\left(E_{\mathrm{F}}, T\right)$ is a linear function of temperature. We attribute this behaviour to the pseudogap, because it was demonstrated that the antinode in underdoped samples is dominated by the pseudogap and does not contribute to the superfluid density ${ }^{15,16}$. This almost perfect scaling behaviour of $W_{\text {pair }}$ with $T_{\text {pair }}$ not only validates our extraction of the pairing component, but it also gives compelling evidence that $T_{\text {pair }}$ is the onset temperature of pairing and that pairing is not the origin of the pseudogap, because the pseudogap persists to the much higher $T^{*}$.

We summarize our results in Fig. 4 in the form of a phase diagram. We compare our data to the detection of phasefluctuating superconductivity by magnetization ${ }^{6}$, the Nernst effect ${ }^{5}$, NMR (ref. 26) and specific heat ${ }^{27}$. We note the reasonable agreement between these probes and angle-resolved photoemission spectroscopy (ARPES). Note that ARPES reports slightly different temperatures, because the other probes are sensitive to a weighted average over the Fermi surface, whereas with ARPES we can extract these directly for the antinodal areas, where this temperature is expected to be highest. We note that specific-heat results ${ }^{27}$ were carried out using Bi2212 samples and exhibit slightly higher pairing fluctuation temperatures, in agreement with our Bi2212 results in Fig. 1. The pairing temperature $\left(T_{\text {pair }}\right)$ (shown in Fig. $4 \mathrm{~b}$ ) increases steadily from the overdoped side of the phase diagram towards optimal doping. For dopings lower than optimal, it levels off at $\sim 120 \mathrm{~K}$. This behaviour contrasts with that of the pseudogap 


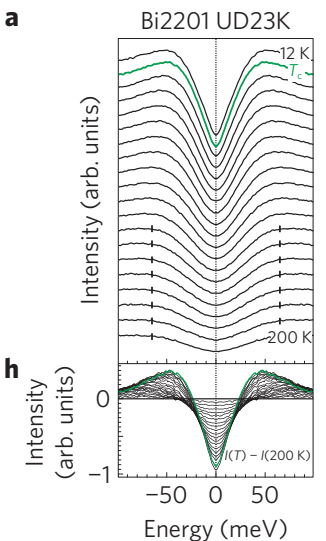

o

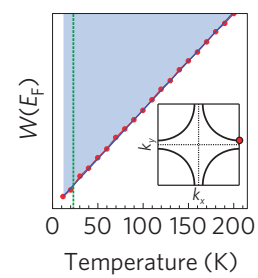

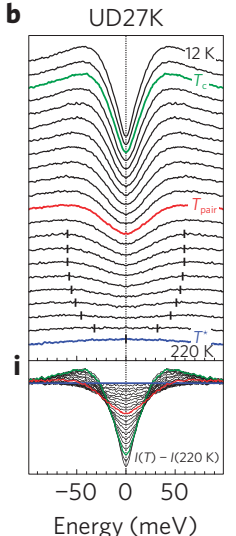
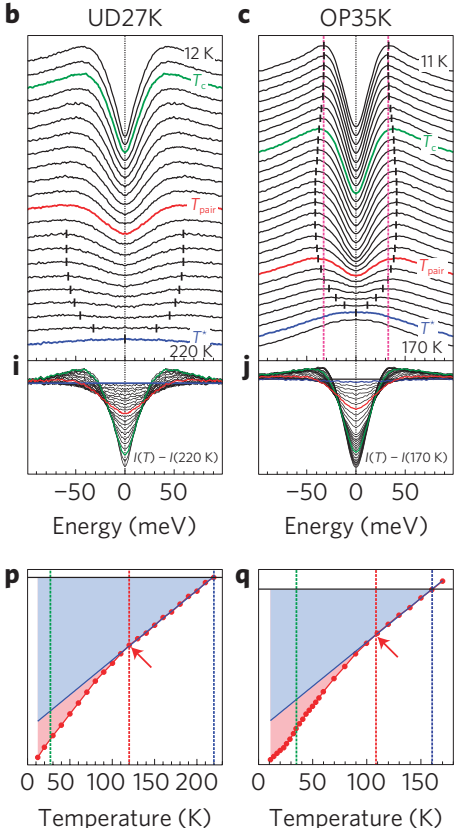
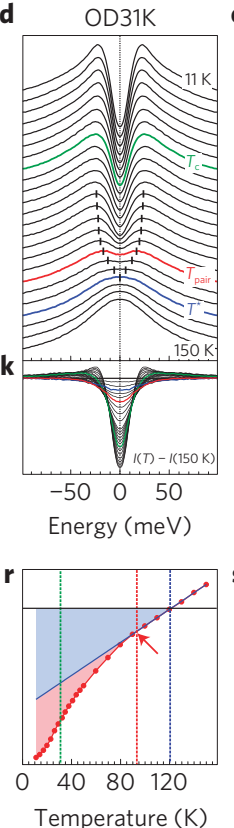
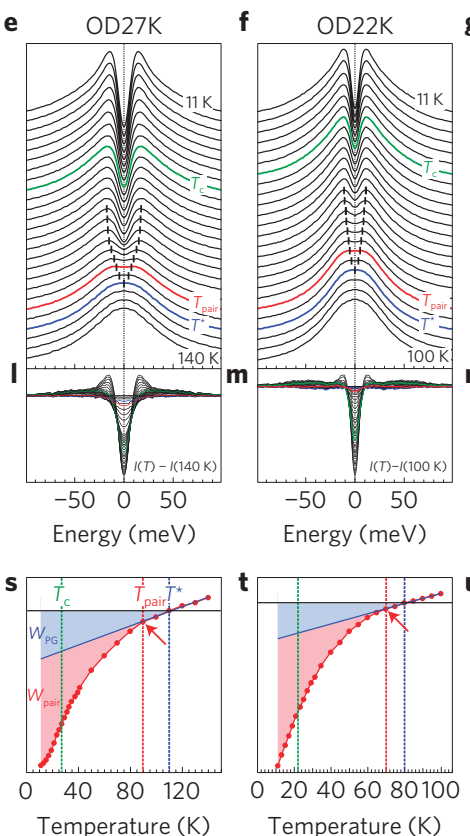
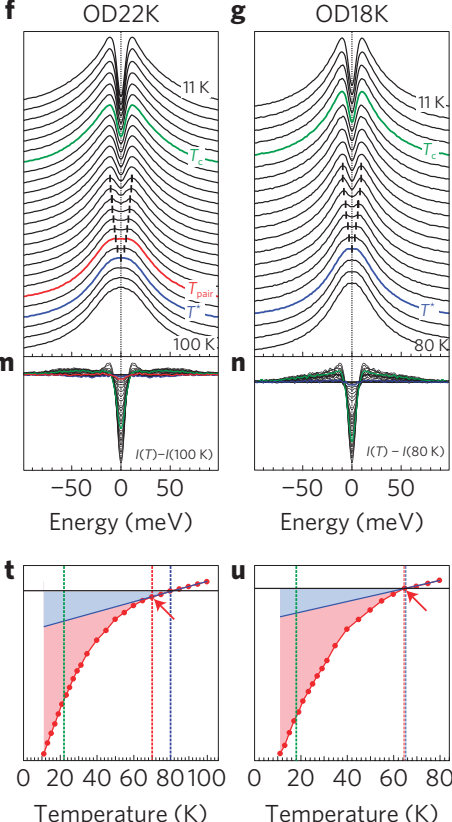

Energy (meV)

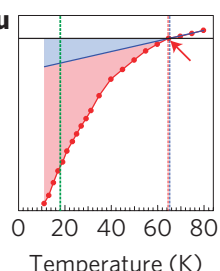

Figure 2 | Doping and temperature dependence of spectral weight at the Fermi energy. a-g, Symmetrized EDCs at various temperatures measured at the antinode (marked by the red point on the Fermi surface shown in the inset of $\mathbf{o}$ ) in Bi2201 over a wide range of doping from underdoping (left) to overdoping (right). An offset is used for clarity. $\mathbf{h}-\mathbf{n}$, Difference spectra. The spectrum at the highest temperature is subtracted from each of the spectra in a-g. o-u, Temperature dependence of the spectral weight close to $E_{F}, W\left(E_{\mathrm{F}}\right)$, obtained by integrating the spectra in $\mathbf{h}-\mathbf{n}$ within the energy resolution window (10 meV) about $E_{\mathrm{F}}$ (see Fig. 1b). The three temperatures, $T_{\mathrm{c}}$ (green), $T_{\text {pair }}$ (red) and $T^{*}$ (blue), are indicated with dashed lines. The pairing weight $\left(W_{\text {pair, }}\right.$ red area) and the pseudogap weight ( $W_{\mathrm{PG}}$, blue area) are separated by a line extrapolated from the linear behaviour of $W\left(E_{\mathrm{F}}\right)$ at high temperatures. Error bars in EDC curves (a-n) are statistical and reflected by noise in the data. Maximum measurement errors $\pm 1 \%$ in $\mathbf{0}-\mathbf{u}$ are due to uncertainty in the normalization and are smaller than data markers.
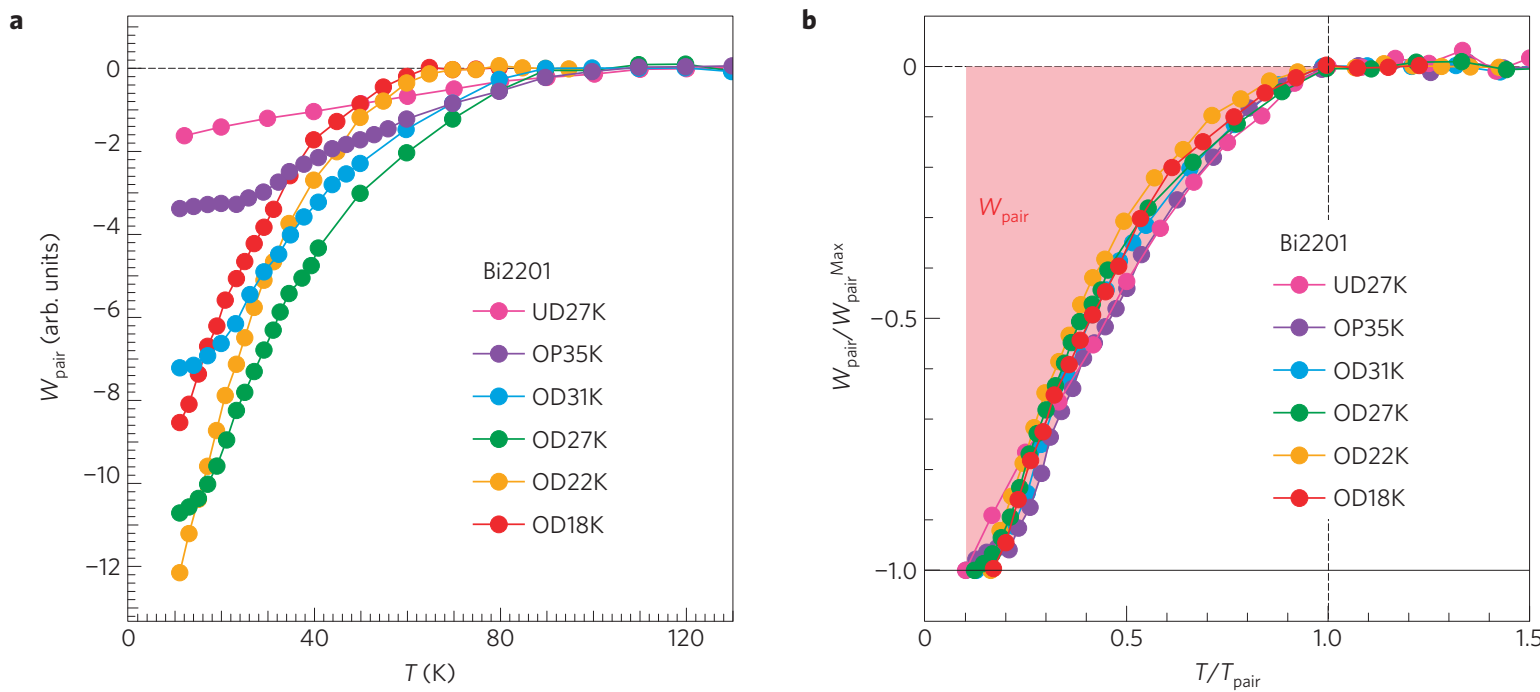

Figure $\mathbf{3}$ | Universal scaling behaviour of the pairing spectral weight. a, Temperature dependence of $\left(W_{\text {pair }}\right)$ for all samples extracted by subtracting the extrapolated $W_{P G}$ line from the $W\left(E_{F}\right)$ curves in Fig. $2 \mathrm{~h}-\mathrm{n} . \mathbf{b}, W_{\text {pair }}$ from a scaled with the pairing temperature $\left(T_{\text {pair }}\right)$ and the maximum value at the lowest temperature $\left(W_{\text {pair }}{ }^{M a x}\right)$. Maximum measurement errors $\pm 1 \%$ in $\mathbf{a}$ and $\mathbf{b}$ are due to uncertainty in the normalization and are smaller than the data markers.

temperature $\left(T^{*}\right)$ (Fig. 4c), which monotonically increases up to the lowest doping. We note that the pairing temperature of Bi2201 is similar to that of Bi2212 (see Fig. 1d), despite the large difference of $T_{\mathrm{c}}$. This strongly suggests that the onset temperature of pairing $(120-150 \mathrm{~K})$ is universal and similar to the highest achieved superconducting temperature in the cuprates. Quantitative analysis of the very detailed ARPES data presented here provides clear evidence for a spectroscopic temperature scale $T_{\text {pair }}$, distinct from $T_{\mathrm{c}}$ and $T^{*}$. It demonstrates that pairing and the pseudogap are two fundamentally different states. The competition between the two states may play a key role in the determination of $T_{\mathrm{c}}$, where bulk superconductivity is established.

The remaining open question is the origin of the pseudogap. Recent results from scanning tunnelling microscopy (STM) suggested ${ }^{23,28}$ that the pseudogap is associated with a 'checkerboard' pattern ${ }^{29}$. Although direct evidence for this (for example, vanishing of the 'checkerboard' pattern at $T^{*}$ ) is still missing, our data seem to support this scenario. Figure $3 \mathrm{~d}$ of ref. 30 shows the relation 


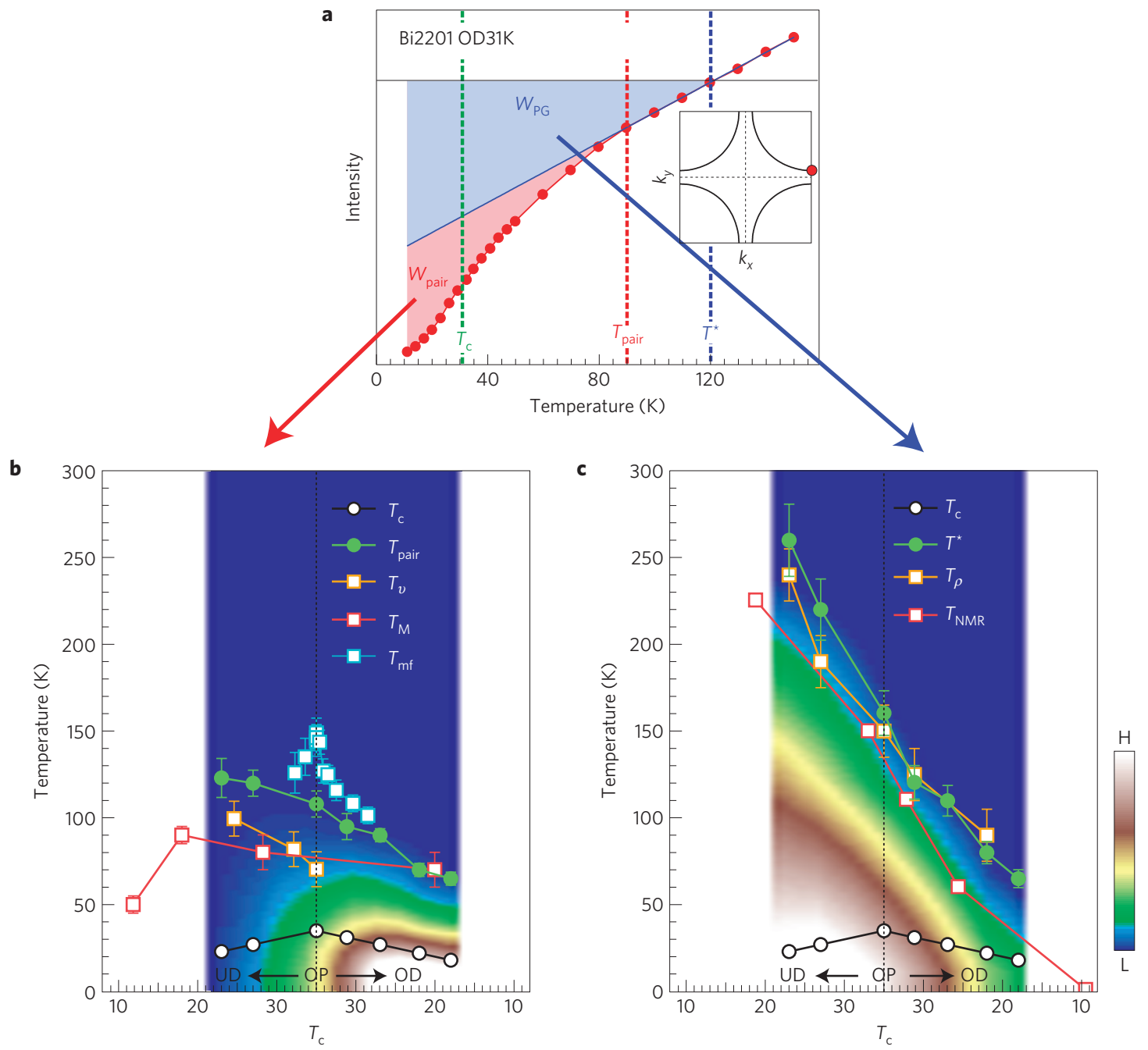

Figure 4 | Phase diagrams obtained using pairing and pseudogap spectral weights. a, $W\left(E_{\mathrm{F}}\right)$ versus $T$ plots for OD31K, the same as Fig. $2 \mathrm{r}$. b, Phase diagram obtained using the pairing spectral weight ( $W_{\text {pair, }}$ red area in $\left.\mathbf{a}\right)$. The onset temperature of pair formation estimated from our ARPES data $\left(T_{\text {pair }}\right)$, the onset temperature of the Nernst effect ${ }^{5}\left(T_{v}\right)$, the diamagnetic effect ${ }^{6}\left(T_{M}\right)$ and specific heat ${ }^{27}\left(T_{\text {mf }}\right)$ are plotted for comparison. As the specific-heat measurements were carried out using Bi2221, the horizontal axis for $T_{\mathrm{mf}}$ plots was scaled by the ratio of $T_{\mathrm{c}}$ values for Bi2212 and Bi2201. Note that $T_{\text {pair }}$ for UD23K was extracted from data around $13^{\circ}$ off the antinode because both quasiparticle peaks and $W_{\text {pair }}$ are very small at the antinode. $\mathbf{c}$, Phase diagram obtained using the pseudogap spectral weight ( $W_{P G}$, blue area in $\left.\mathbf{a}\right)$. The pseudogap temperature estimated from our ARPES data ( $\left.T^{*}\right)$, our resistivity results ( $T_{\rho}$, see Supplementary Fig. S1) and NMR ( $T_{N M R}$; ref. 26$)$ are plotted. Error bars in $\mathbf{b}$ represent uncertainty in estimating the temperatures at which deviation from linear behaviour occurs (obtained by interpolation from errors in $W\left(E_{\mathrm{F}}\right)$. Error bars in c represent uncertainty in estimating the temperature at which the pseudogap closes (that is, two peaks merge into a single peak at $E_{\mathrm{F}}$. Experimental errors in a are the same as in Fig. 1d.

between a 'checkerboard' wave vector and pseudogap energy. The average binding energies (represented by black data points), for which the 'checkerboard' is observed in STM measurements agrees with the values of the pseudogap we find for the same samples with ARPES. There is also no STM evidence for a 'checkerboard' pattern in overdoped samples, where the pseudogap is absent.

The fact that we observe perfect scaling of $W_{\text {pair }}$ with $T_{\text {pair }}$, regardless of the doping, implies that the only two relevant energy scales are the order parameter of the pseudogap state and the magnitude of the pairing potential, making the existence of the three temperature scales $T_{\mathrm{c}}<T_{\text {pair }}<T^{*}$, a universal aspect of underdoped cuprates. The observed scaling behaviour with respect to $T_{\text {pair }}$ indicates a breakdown of the classical picture of phase fluctuations in the cuprates and gives an important clue as to the nature of the Cooper-pair fluctuations: it is not sufficient to solely consider phase fluctuations of the pairing field. $T_{\text {pair }}$ is the temperature where the amplitude of the pairing field melts, that is, where the strength of incoherent pairing disappears. The emergence of $T_{\text {pair }}$ as the relevant temperature of the scaling requires that the pair-amplitude and phase fluctuation are equally crucial below $T_{\text {pair }}$. A pairing interaction that is in the extreme strong-coupling limit, reminiscent of Mott physics, was shown to lead to simultaneous amplitude and pair modes that separate coherent and local pairing ${ }^{30}$.

\section{Methods}

Optimally doped $\mathrm{Bi}_{2} \mathrm{Sr}_{2} \mathrm{CaCu}_{2} \mathrm{O}_{8+\delta}$ (Bi2212) single crystals with $T_{\mathrm{c}}=90 \mathrm{~K}$ $(\mathrm{OP} 90 \mathrm{~K})$ and $(\mathrm{Bi}, \mathrm{Pb})_{2}(\mathrm{Sr}, \mathrm{La})_{2} \mathrm{CuO}_{6+\delta}(\mathrm{Bi} 2201)$ single crystals with various $T_{c}$ values were grown by the conventional floating-zone technique (see Supplementary Information on the sample characterization). To precisely analyse the ARPES spectra, we partially substituted $\mathrm{Pb}$ for $\mathrm{Bi}$ for all Bi2201 samples, and suppressed the modulation in the $\mathrm{BiO}$ plane, which usually contaminates the ARPES signal. ARPES data were acquired using a laboratory-based system consisting of a Scienta SES2002 electron analyser and a GammaData helium ultraviolet lamp. All data were acquired using the $\mathrm{HeI}$ line with a photon energy of $21.2 \mathrm{eV}$. The angular resolution was $0.13^{\circ}$ and $\sim 0.5^{\circ}$ along and perpendicular to the direction of the analyser slits, respectively. The energy resolution was set at $\sim 10 \mathrm{meV}-$ confirmed by measuring the energy width between the $90 \%$ and $10 \%$ of the Fermi edge 
from the same Au reference. Custom-designed refocusing optics enabled us to accumulate high-statistics spectra in a short time without effects of sample surface ageing. In the analysis we used symmetrized EDCs normalized to the total area over the whole energy range $(-0.4 \mathrm{eV} \leq E \leq 0.4 \mathrm{eV})$ for each spectrum. We verified that the choice of normalization scheme does not affect the results of our analysis (see Supplementary Information on the details). The results were reproduced on several samples and on temperature cycling.

Received 21 May 2010; accepted 15 October 2010; published online 5 December 2010

\section{References}

1. Warren, W. W. et al. Cu spin dynamics and superconducting precursor effects in planes above $T_{\mathrm{c}}$ in $\mathrm{YBa}_{2} \mathrm{Cu}_{3} \mathrm{O}_{6.7}$. Phys. Rev. Lett. 62, 1193-1196 (1989).

2. Takigawa, M. et al. $\mathrm{Cu}$ and $\mathrm{O}$ NMR-studies of the magnetic properties of $\mathrm{YBa}_{2} \mathrm{Cu}_{3} \mathrm{O}_{6.63}\left(T_{\mathrm{c}}=62 \mathrm{~K}\right)$. Phys. Rev. B 43, 247-257 (1991).

3. Homes, C. C., Timusk, T., Liang, R., Bonn, D. A. \& Hardy, W. N. Optical conductivity of $c$-axis oriented $\mathrm{YBa}_{2} \mathrm{Cu}_{3} \mathrm{O}_{6.70}$-evidence for a pseudogap. Phys. Rev. Lett. 71, 1645-1648 (1993).

4. Emery, V. J. \& Kivelson, S. A. Importance of phase fluctuations in superconductors with small superfluid density. Nature 374, 434-437 (1995).

5. Wang, Y. et al. Onset of the vortexlike Nernst signal above $T_{c}$ in $\mathrm{La}_{2-x} \mathrm{Sr}_{x} \mathrm{CuO}_{4}$ and $\mathrm{Bi}_{2} \mathrm{Sr}_{2-y} \mathrm{La}_{y} \mathrm{CuO}_{6}$. Phys. Rev. B 64, 224519 (2001).

6. Lu, Li et al. Diamagnetism and Cooper pairing above $T_{\mathrm{c}}$ in cuprates. Phys. Rev. $B$ 81, 054510 (2010).

7. Lee, J. et al. Spectroscopic fingerprint of phase-incoherent superconductivity in the underdoped $\mathrm{Bi}_{2} \mathrm{Sr}_{2} \mathrm{CaCu}_{2} \mathrm{O}_{8+\delta}$. Science 325, 1099-1103 (2009).

8. Corson, J., Mallozzi, R., Orenstein, J., Eckstein, J. N. \& Bozovic, I. Vanishing of phase coherence in underdoped $\mathrm{Bi}_{2} \mathrm{Sr}_{2} \mathrm{CaCu}_{2} \mathrm{O}_{8+\delta}$. Nature 398, 221-223 (1999).

9. Bergeal, N. et al. Pairing fluctuations in the pseudogap state of copper-oxide superconductors probed by the Josephson effect. Nature Phys. $\mathbf{4}$ 608-611 (2008).

10. Panagopoulos, C. \& Xiang, T. Relationship between the superconducting energy gap and the critical temperature in high- $T_{\mathrm{c}}$ superconductors. Phys. Rev. Lett. 81, 2336-2339 (1998).

11. Tanaka, K. et al. Distinct Fermi-momentum-dependent energy gaps in deeply underdoped Bi2212. Science 314, 1910-1913 (2006).

12. Le Tacon, M. et al. Two energy scales and two distinct quasiparticle dynamics in the superconducting state of underdoped cuprates. Nature Phys. 2, 537-543 (2006).

13. Kondo, T., Takeuchi, T., Kaminski, A., Tsuda, T. \& Shin, S. Evidence for two energy scales in the superconducting state of optimally doped $(\mathrm{Bi}, \mathrm{Pb})_{2}(\mathrm{Sr}, \mathrm{La})_{2} \mathrm{CuO}_{6+\delta}$. Phys. Rev. Lett. 98, 267004 (2007).

14. Boyer, M. C. et al. Imaging the two gaps of the high-temperature superconductor $\mathrm{Bi}_{2} \mathrm{Sr}_{2} \mathrm{CuO}_{6+x}$. Nature Phys. 3, 802-806 (2007).

15. Khasanov, R. et al. Evidence for competition between the superconducting and the pseudogap state in $(\mathrm{BiPb})_{2}(\mathrm{SrLa})_{2} \mathrm{CuO}_{6+x}$ from muon-spin rotation experiments. Phys. Rev. Lett. 101, 227002 (2008).

16. Kondo, T., Khasanov, R., Takeuchi, T., Schmalian, J. \& Kaminski, A. Competition between the pseudogap and superconductivity in the high- $T_{\mathrm{c}}$ copper oxides. Nature 457, 296-300 (2009).
17. Ding, H. et al. Spectroscopic evidence for a pseudogap in the normal state of underdoped high- $T_{\mathrm{c}}$ superconductors. Nature 382, 51-54 (1996).

18. Loeser, A. G. et al. Excitation gap in the normal state of underdoped $\mathrm{Bi}_{2} \mathrm{Sr}_{2} \mathrm{CaCu}_{2} \mathrm{O}_{8+x}$. Science 273, 325-329 (1996).

19. Meng, J. et al. Motonic d-wavesupercondcuting gap in optimally-doped $\mathrm{Bi}_{2} \mathrm{Sr}_{1.6} \mathrm{La}_{0.4} \mathrm{CuO}_{6+\delta}$ superconductor by laser-based angle-resolved photoemission spectroscopy. Phys. Rev. B 79, 024514 (2009).

20. Nakayama, K. et al. Evolution of a pairing-induced pseudogap from the superconducting gap of $\mathrm{Bi}_{2} \mathrm{Sr}_{2} \mathrm{CuO}_{6+\delta}$. Phys. Rev. Lett. 102, 227006 (2009).

21. Pan, S. H. et al. Microscopic electronic inhomogeneity in the high- $T_{c}$ superconductor $\mathrm{Bi}_{2} \mathrm{Sr}_{2} \mathrm{CaCu}_{2} \mathrm{O}_{8+x}$. Nature 413, 282-285 (2001).

22. Gomes, K. K. et al. Visualizing pair formation on the atomic scale in the high- $T_{\mathrm{c}}$ superconductor $\mathrm{Bi}_{2} \mathrm{Sr}_{2} \mathrm{CaCu}_{2} \mathrm{O}_{8+\delta}$. Nature 447, 569-572 (2007).

23. Wise, W. D. et al. Charge-density-wave origin of cuprate checkerboard visualized by scanning tunnelling microscopy. Nature Phys. 4, 696-699 (2008).

24. Norman, M. R. et al. Destruction of the Fermi surface in underdoped high- $T_{c}$ superconductors. Nature 392, 157-160 (1998).

25. Wei, J. et al. Superconducting coherence peak in the electronic excitations of a single layer cuprate superconductor $\mathrm{Bi}_{2} \mathrm{Sr}_{1.6} \mathrm{La}_{0.4} \mathrm{CuO}_{6+\delta}$. Phys. Rev. Lett. 101, 097005 (2008).

26. Zheng, G., Kuhns, P. L., Reyes, A. P., Liang, B. \& Lin, C. T. Critical point and the nature of the pseudogap of single-layered copper-oxide $\mathrm{Bi}_{2} \mathrm{Sr}_{2-x} \mathrm{La}_{x} \mathrm{CuO}_{6+\delta}$ superconductors. Phys. Rev. Lett. 94, 047006 (2005).

27. Tallon, J. L. et al. Fluctuations and $T_{\mathrm{c}}$ reduction in cuprate superconductors. Preprint at http://arxiv.org/abs/0908.4428 (2009).

28. Wise, W. D. et al. Imaging nanoscale Fermi-surface variations in an inhomogeneous superconductor. Nature Phys. 5, 213-216 (2009).

29. Hanaguri, T. et al. A 'checkerboard' electronic crystal state in lightly hole-doped $\mathrm{Ca}_{2-x} \mathrm{Na}_{x} \mathrm{CuO}_{2} \mathrm{Cl}_{2}$. Nature 430, 1001-1005 (2004).

30. Chubukov, A. V. \& Schmalian, J. Superconductivity due to massless boson exchange in the strong-coupling limit. Phys. Rev. B 72, 174520 (2005).

\section{Acknowledgements}

We thank A. Millis, C. Varma and M. Norman for useful discussions. This work was supported by Basic Energy Sciences, US DOE. The Ames Laboratory is operated for the US DOE by Iowa State University under Contract No. W-7405-ENG-82. Work at Brookhaven is supported by the US DOE under Contract No. DE-AC02-98CH10886. J.S.W. and Z.J.X. are supported by the Center for Emergent Superconductivity, an Energy Frontier Research Center funded by the US DOE, Office of Science.

\section{Author contributions}

T.K. and A.K. designed the experiment. T.K., Y.H., T.T., J.S.W., Z.J.X. and G.G. grew the high-quality single crystals. T.K. and A.D.P. acquired the experimental data and T.K. carried out the data analysis. T.K., A.K. and J.S. wrote the manuscript. All authors discussed the results and commented on the manuscript.

\section{Additional information}

The authors declare no competing financial interests. Supplementary information accompanies this paper on www.nature.com/naturephysics. Reprints and permissions information is available online at http://npg.nature.com/reprintsandpermissions. Correspondence and requests for materials should be addressed to T.K. or A.K. 